\title{
Acyclic Sets of Linear Orders via the Bruhat Orders
}

\author{
Ádám Galambos \\ Kellogg School of Management \\ Northwestern University \\ a-galambos@kellogg.northwestern.edu
}

\author{
Victor Reiner \\ Department of Mathematics \\ University of Minnesota \\ reiner@math.umn.edu
}

February 28, 2005

\begin{abstract}
We use Ziegler's results [16] on the higher Bruhat orders to show that Abello's acyclic sets of linear orders [1] can be described as the permutations of equivalence classes of maximal reduced decompositions. This allows us to strengthen Abello's structural result: we show that acyclic sets arising from this construction are distributive sublattices of the weak Bruhat order. Fishburn's "alternating scheme" is the set of permutations of one such equivalence class of maximal reduced decompositions. Any acyclic set that arises in this way can be represented by an arrangement of pseudolines, and we use this representation to derive a formula enumerating the "alternating scheme."
\end{abstract}

\section{Introduction}

Majority voting is one of the most commonly accepted and widely practiced methods for aggregating preferences. It is well-known that social preferences determined by majority voting on every pair of alternatives may be intransitive. On the other hand, if voters' preferences are restricted to lie within certain domains, the problem of intransitivity can 
be avoided. Social choice theorists have found several such domain restrictions [14, 12], and this has led to the question: Given $n$ alternatives, what is the cardinality of the largest domain of linear orders that still guarantees transitive social preferences under pairwise majority voting? ${ }^{1}$ Such a domain is called an acyclic set of linear orders. ${ }^{2}$

The question of finding maximum cardinality acyclic sets has proven to be one of the most difficult combinatorial questions in social choice theory [7]. While several upper and lower bounds have been found, and others conjectured $[1,6,11,7]$, the only successful attempt to study the structure of "large" acyclic sets is Abello's [1]. ${ }^{3}$ He showed that a maximal chain in the "weak Bruhat order" forms an acyclic set. Moreover, any maximal acyclic set containing a maximal chain is an upper semimodular sublattice of the weak Bruhat order ([1], Theorem 3.3). Our contribution is describing explicitly these maximal acyclic sets containing a maximal chain in the weak Bruhat order, and proving a slightly strengthened version of Abello's Theorem 3.3 in a transparent way. We also show that Fishburn's "alternating scheme" $[6,7]$ is the maximal acyclic set containing a particular chain in the weak Bruhat order. Our approach is based on results on the higher Bruhat orders $[16,5]$. Placing Fishburn's "alternating scheme" in this formal framework allows us to derive an explicit formula enumerating it.

In section 2 we define the combinatorial objects we will use, and discuss the relationships among them. In section 3 we describe explicitly the maximal acyclic sets that contain a maximal chain in the weak Bruhat order, and show how the "alternating scheme" fits in that framework. We derive the formula enumerating the alternating scheme in section 3.1.

\footnotetext{
${ }^{1}$ We assume throughout that the number of voters is at least 3 . This question can be posed without reference to the number of voters because if a preference profile results in intransitive social preferences under majority voting, then it contains 3 preferences that would, by themselves, result in such intransitivity $[12]$.

${ }^{2}$ Henceforth "acyclic sets."

${ }^{3}$ An early paper by Chameni-Nembua [3] seems to have anticipated some of Abello's and our results.
} 


\section{Definitions}

Let $\mathcal{R}$ be the set of all total, transitive, reflexive and antisymmetric binary relations (i.e. linear orders) on the finite set $A$ of social alternatives. The finite set of agents will be denoted by $I$, and their preferences $\left(R_{i}\right)_{i \in I}$ are from $\mathcal{R}$. Under majority voting, social preferences $R^{M}$ are defined by: for all $a, b \in A$

$$
a R^{M} b \Longleftrightarrow\left|\left\{i \in I: a R_{i} b\right\}\right| \geq\left|\left\{i \in I: b R_{i} a\right\}\right| \text {. }
$$

For simplicity, we identify the $n$-set $A$ with the set $[n]:=\{1,2, \ldots, n\}$ under an arbitrary, fixed ordering of $A$, and we represent individual preferences as permutations of $[n]$. A permutation $\pi:[n] \rightarrow[n]$ will be identified with the linear order

$$
\pi^{-1}(1)>\pi^{-1}(2)>\cdots>\pi^{-1}(n),
$$

and will be written as $\pi^{-1}(1) \pi^{-1}(2) \cdots \pi^{-1}(n)$. The set of permutations is denoted by $S_{n}$.

Definition 2.1 A set $T \subseteq S_{n}$ is acyclic if for all $i, j, k \in[n]$, at most two of the orders $i j k, j k i, k i j$ appear as a restriction to $\{i, j, k\}$ of some order in $T$.

Ward [14] introduced this condition as "latin squarelessness," 4 and showed that with an odd number of voters it guarantees transitive majority. Sen [12] introduced this condition for the setting where indifference in individual preferences is allowed, and called it the "assumption of value-restricted preferences." He showed that in that setting it guarantees transitive majority. While a profile of preferences that is not acyclic may produce a transitive social preference under majority voting, acyclicity is clearly necessary in the following sense: if a domain of preferences always produces a transitive majority regardless of how many agents have each particular preference relation, then this domain must be acyclic. ${ }^{5}$

\footnotetext{
${ }^{4}$ Ward comments on the term: "awkward, but there is a suggestive rythmic harmony with its predecessor, single peakedness." [14]

${ }^{5}$ Notice that "acyclicity" is the property of a set of preferences, not of a profile of preferences. It is for this reason that we do not discuss restrictions on the parity of the number of agents, although, for any particular preference profile, it is important.
} 
Abello [1] used the structure imposed on the set $S_{n}$ of linear orders by the "weak Bruhat order" to construct acyclic sets. Since then significant work has been done on the "higher Bruhat orders" $[9,10,16,5]$. Some of these results are central to our approach, so we present them as we give the following definitions.

For any permutation $\pi \in S_{n}$, let

$$
\operatorname{inv}(\pi):=\{\{i, j\}: i<j \text { and } \pi(i)>\pi(j)\}
$$

denote the inversion set of $\pi$. For example, inv $(2143)=\{\{1,2\},\{3,4\}\}$. Let $B(n, 1):=$ $\left\{\operatorname{inv}(\pi): \pi \in S_{n}\right\}$.

Definition 2.2 The weak Bruhat order $\mathbb{B}(n, 1)$ is the partial order on $B(n, 1)$ defined by the transitive closure of single step set inclusion. That is, for any two permutations $\sigma$ and $\pi, \quad i n v(\sigma)$ covers $i n v(\pi)$ if and only if $i n v(\pi) \subseteq i n v(\sigma)$ and $|i n v(\pi)|+1=|\operatorname{inv}(\sigma)|$.

The weak Bruhat order is a lattice [16, Theorem 4.4], and it is the same as $B_{\subseteq}(n, 1)$, the set $B(n, 1)$ partially ordered by set inclusion [15, Prop. 2.1].

Since every permutation of $[n]$ can be uniquely identified by its inversion set, the weak Bruhat order can also be viewed as a partial order on permutations. For example, part b) of Figure 1 shows the weak Bruhat order $\mathbb{B}(3,1)$, while part a) shows it as a partial order on $S_{3}$.

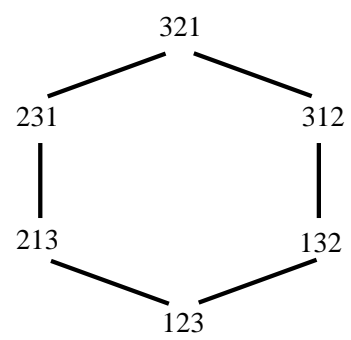

a)

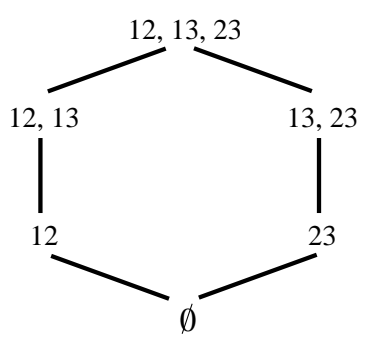

b)

Figure 1: The weak Bruhat order $\mathbb{B}(3,1)$

A maximal chain in $\mathbb{B}(n, 1)$ is a set of permutations $\left\{\pi_{1}, \pi_{2}, \ldots, \pi_{l}\right\}$ such that $\pi_{i}$ covers $\pi_{i+1}$ for $i=1, \ldots, l-1$, and $\pi_{l}$ is the identity while $\pi_{1}$ is its reverse. A maximal chain can 
be identified by the sequence of transpositions that generates it. For example, we could identify the maximal chain

$$
1234,2134,2314,2341,3241,3421,4321
$$

by saying: transpose the first and the second element, then the second and the third, etc. Since we always transpose adjacent elements, naming just the position of the left element to be transposed suffices. Denoting the transpositions of the $i$ th and the $i+1$ st element by $s_{i}$, we could describe the above sequence as

$$
1234 \stackrel{s_{1}}{\longrightarrow} 2134 \stackrel{s_{2}}{\longrightarrow} 2314 \stackrel{s_{3}}{\longrightarrow} 2341 \stackrel{s_{1}}{\longrightarrow} 3241 \stackrel{s_{2}}{\longrightarrow} 3421 \stackrel{s_{1}}{\longrightarrow} 4321 .
$$

In fact, we may even omit the permutations — we could always recover them from the sequence of transpositions: $s_{1} s_{2} s_{3} s_{1} s_{2} s_{1}$. We assume, implicitly, that the sequence of permutations always starts with the identity. Such a sequence of increasing adjacent transpositions $s_{i}$, resulting in the reverse of the identity, is called a maximal reduced decomposition $\left[2\right.$, Section 6.4]. ${ }^{6}$ The permutations visited by a maximal reduced decomposition are the permutations in the maximal chain in the weak Bruhat order it corresponds to. For example, the permutations visited by $s_{1} s_{2} s_{3} s_{1} s_{2} s_{1}$ above are

$$
\begin{aligned}
i d & =1234 \\
s_{1} & =2134 \\
s_{1} s_{2} & =2314 \\
s_{1} s_{2} s_{3} & =2341 \\
s_{1} s_{2} s_{3} s_{1} & =3241 \\
s_{1} s_{2} s_{3} s_{1} s_{2} & =3421 \\
s_{1} s_{2} s_{3} s_{1} s_{2} s_{1} & =4321 .
\end{aligned}
$$

\footnotetext{
${ }^{6}$ Maximal because it starts with the identity and ends with its reverse, and reduced because it has minimum length (namely $\left(\begin{array}{l}n \\ 2\end{array}\right)$ ) among all the ones that start with the identity and end with its reverse. The latter requirement is equivalent to allowing only increasing transpositions: $\ldots i j \ldots \rightarrow \ldots j i \ldots$ with $i<j$.
} 
A maximal reduced decomposition can also be viewed as a permutation of $\left(\begin{array}{c}{[n]} \\ 2\end{array}\right)$, the 2 -subsets of $[n]$. Since it starts with the identity and ends with its reverse, every pair must be transposed, and since it is "reduced," every pair must be transposed exactly once. The sequence of these transpositions gives us a permutation of (unordered) pairs. In fact, a structure that generalizes the weak Bruhat order can be imposed on the set of all such permutations of pairs, or, generally, $k$-subsets of $[n]$. More precisely, the higher Bruhat order $\mathbb{B}(n, k)$ is defined on the "inversion sets" of certain permutations of $\left(\begin{array}{c}{[n]} \\ k\end{array}\right)$.

Definition 2.3 [16] A permutation of $\left(\begin{array}{c}{[n]} \\ k\end{array}\right)$ is admissible if the $k$-subsets of any $k+1$ subset of $[n]$ appear either in lexicographic or in reversed lexicographic order in it. The inversion set $\operatorname{inv}(\rho) \subseteq\left(\begin{array}{c}{[n]} \\ k+1\end{array}\right)$ of an admissible permutation $\rho$ is the set of $k+1$-subsets of $[n]$ whose $k$-subsets appear in reversed lexicographic order in $\rho$.

For example, inv $(\{1,2\}\{3,4\}\{1,4\}\{2,4\}\{1,3\}\{2,3\})=\{\{1,3,4\},\{2,3,4\}\}$.

Definition $2.4 \quad[\mathbf{1 6}]$ Let

$$
B(n, k):=\left\{\operatorname{inv}(\pi): \pi \text { is an admissible permutation of }\left(\begin{array}{c}
{[n]} \\
k
\end{array}\right)\right\} .
$$

The higher Bruhat order $\mathbb{B}(n, k)$ is the partial order on $B(n, k)$ defined by the transitive closure of single step set inclusion. ${ }^{7}$

Notice that the order is defined on inversion sets, not on the permutations themselves. While the inversion set (a set of 2 -subsets of $[n]$ ) of a permutation of $[n]$ determines the permutation uniquely, the inversion set (a set of $(k+1)$-subsets of $[n]$ ) of a permutation of $\left(\begin{array}{c}{[n]} \\ k\end{array}\right)$ determines an "equivalence class" of permutations. We now illustrate this for $\mathbb{B}(n, 2)$, the higher Bruhat order we will use below.

A maximal chain in the weak Bruhat order, when viewed as a permutation of 2-subsets, is admissible [16, Lemma 2.4]. Moreover, every admissible permutation of $\left(\begin{array}{c}{[n]} \\ 2\end{array}\right)$ can be naturally identified with a maximal chain in the weak Bruhat order [16, Theorem 4.1(A)][10].

\footnotetext{
${ }^{7}$ The higher Bruhat order $\mathbb{B}(n, 2)$ is the same as $B_{\subseteq}(n, 2)$, the set $B(n, 2)$ partially ordered by set inclusion [4]. Note, however, that the analogous statement does not hold for all $\mathbb{B}(n, k)[16]$. Higher Bruhat orders have been shown to be closely related to other combinatorial structures, such as hyperplane arrangements, tilings, and wiring diagrams.
} 
Since maximal chains in $\mathbb{B}(n, 1)$ can be thought of as maximal reduced decompositions, we have that admissible permutations of $\left(\begin{array}{c}{[n]} \\ 2\end{array}\right)$, maximal chains in $\mathbb{B}(n, 1)$ and maximal reduced decompositions are essentially equivalent objects. How can we identify admissible permutations (or maximal chains in $\mathbb{B}(n, 1)$, or maximal reduced decompositions) that have the same inversion sets and thus are mapped to the same element of $\mathbb{B}(n, 2)$ ? Lemma 2.2 in [16] answers this question: two admissible permutations of $\left(\begin{array}{c}{[n]} \\ 2\end{array}\right)$ have the same inversion sets if, and only if, they are equivalent in the equivalence relation induced by the notion of "elementary equivalence:"

Definition 2.5 $\quad$ 16, Def. 2.1] [10, Def. 2.2] Two admissible permutations of $\left(\begin{array}{c}{[n]} \\ 2\end{array}\right)$ are elementarily equivalent if they differ by an interchange of two disjoint neighbors. ${ }^{8}$

To summarize, we illustrate the correspondences among permutations of 2-subsets, maximal reduced decompositions, and maximal chains in the weak Bruhat order. The weak Bruhat order $\mathbb{B}(4,1)$ is shown in Figure 2. Four maximal chains with their corresponding permutations of $\left(\begin{array}{c}{[n]} \\ 2\end{array}\right)$ and maximal reduced decompositions are shown in Figure 3, which the reader may find a useful reference in later discussions as well. These four permutations of $\left(\begin{array}{c}{[n]} \\ 2\end{array}\right)$ are, in fact, equivalent - they all have $\{\{1,3,4\},\{2,3,4\}\}$ as their inversion set. The union of the four maximal chains is the subposet of $\mathbb{B}(4,1)$ highlighted in Figure 2 . The permutations visited by the four maximal reduced decompositions are the permutations in this highlighted subposet. When a set of maximal reduced decompositions correspond to an equivalence class of permutations of $\left(\begin{array}{c}{[n]} \\ 2\end{array}\right)$, we will call them an equivalence class of maximal reduced decompositions. Theorem 1 below states that the permutations visited by an equivalence class of maximal reduced decompositions form an acyclic set.

Since equivalence classes of maximal reduced decompositions turn out to be the central objects in our analysis, we would like to have a concise representation of them. In fact, an equivalence class of maximal reduced decompositions can be represented in a particularly

\footnotetext{
${ }^{8}$ The Lemma and the Definition are originally formulated for permutations of $\left(\begin{array}{c}{[n]} \\ k\end{array}\right), k \geq 1$, though here we consider only the case $k=2$. In general, the neighbors to be interchanged must have at most $k-2$ common elements.
} 


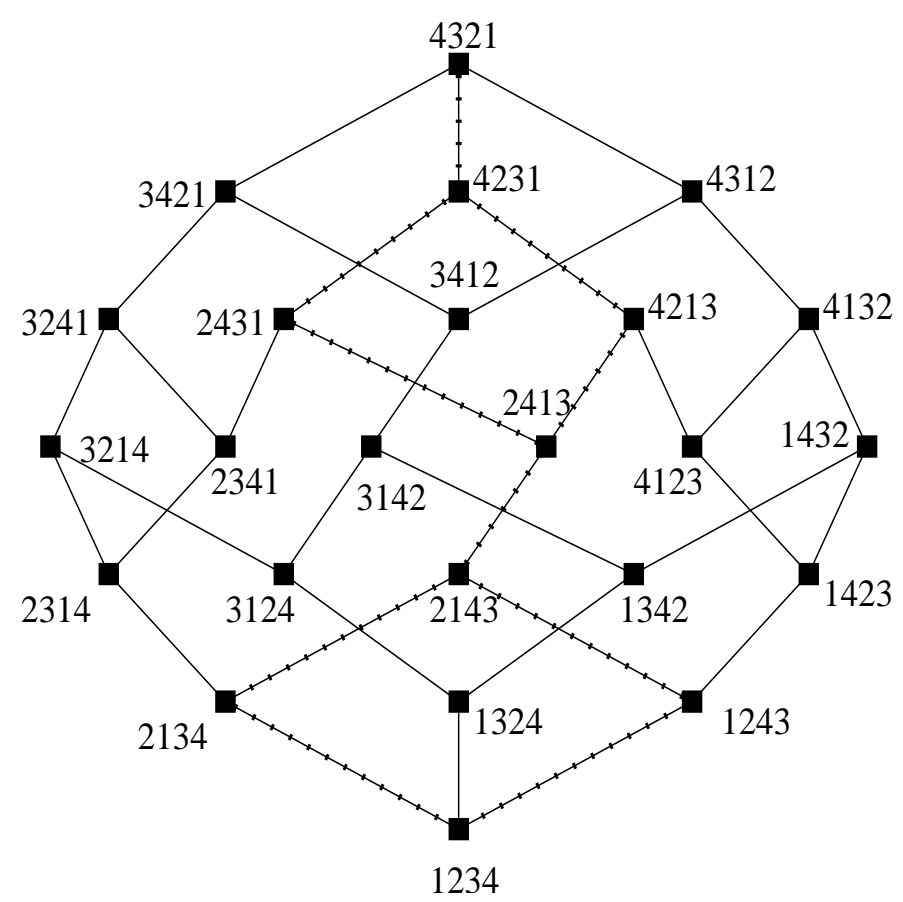

Figure 2: The weak Bruhat order $\mathbb{B}(4,1)$

useful way as an "arrangement of pseudolines."

\subsection{Arrangements of pseudolines}

We illustrate how one can represent an equivalence class of maximal reduced decompositions as an arrangement of pseudolines by demonstrating it on the example used in (6): $s_{1} s_{2} s_{3} s_{1} s_{2} s_{1}$. We associate a "line" with each of the numbers 1,2,3 and 4, and represent the starting permutation, 1234, by placing them in that order. The numbers $4,3,2,1$ on the right indicate that the lines will end up in that order after we carry out all the transpositions. The first transposition, $s_{1}$, corresponds to crossing the first and second lines (see Figure 5 on p. 11). The next transposition is $s_{2}$, so we cross the second and the third lines. ${ }^{9}$ Continuing this way, we cross the third and fourth lines to represent $s_{3}$ (see Figure ??). In general, for $s_{i}$ we cross the $i$ th and $i+1$ st line from the top. After we carry

\footnotetext{
${ }^{9}$ Notice that we did not cross the line that is labelled "2," but, rather, the second line from the top (with the third).
} 


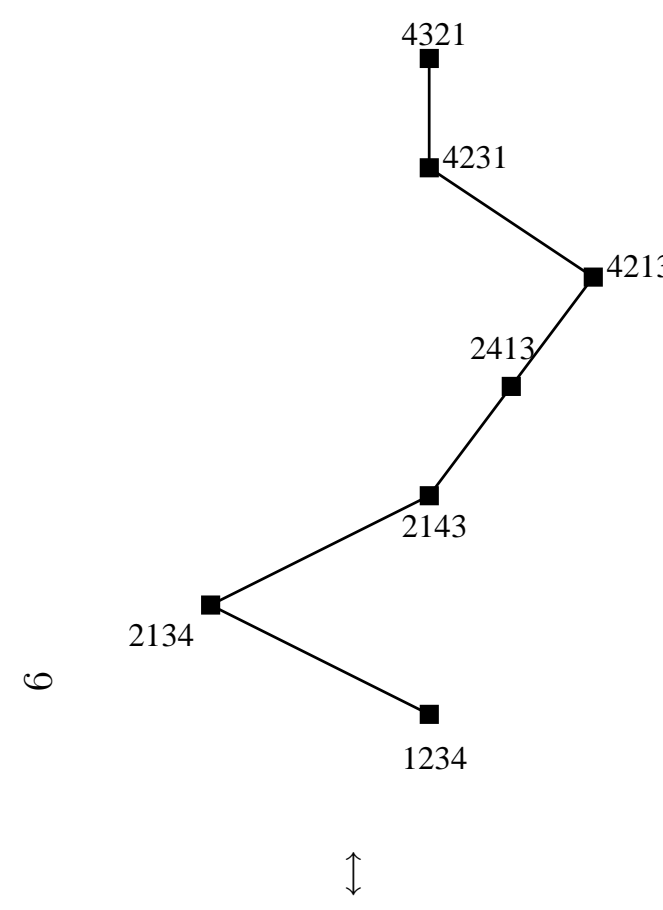

$\{1,2\}\{3,4\}\{1,4\}\{2,4\}\{1,3\}\{2,3\}$

$\uparrow$
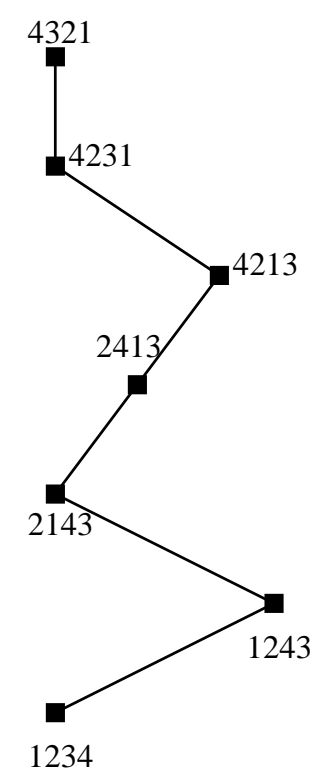

$\uparrow$

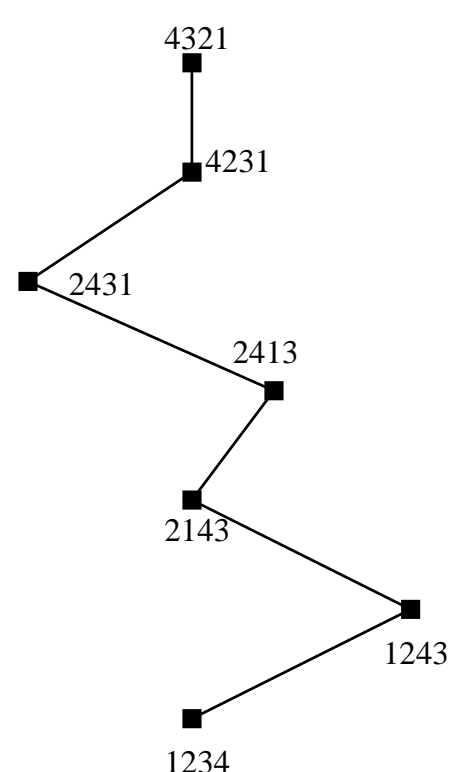

$\uparrow$

$\{3,4\}\{1,2\}\{1,4\}\{1,3\}\{2,4\}\{2,3\}$

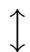

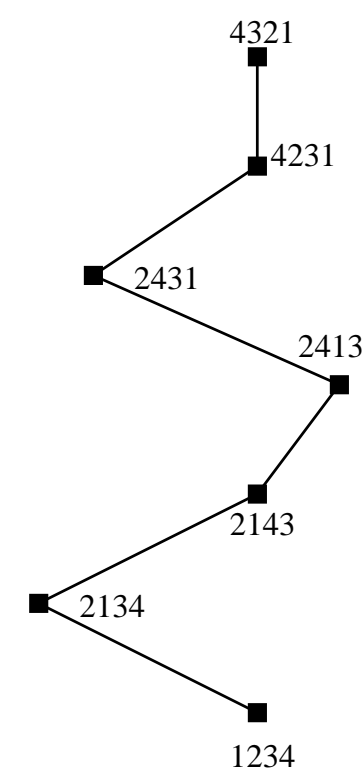

$\uparrow$

$\{1,2\}\{3,4\}\{1,4\}\{1,3\}\{2,4\}\{2,3\}$

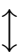

$s_{1} s_{3} s_{2} s_{1} s_{3} s_{2}$

$s_{3} s_{1} s_{2} s_{1} s_{3} s_{2}$

$s_{3} s_{1} s_{2} s_{3} s_{1} s_{2}$

$s_{1} s_{3} s_{2} s_{3} s_{1} s_{2}$

Figure 3: The correspondences among permutations of $\left(\begin{array}{c}{[n]} \\ 2\end{array}\right)$, maximal chains, and maximal reduced decompositions 


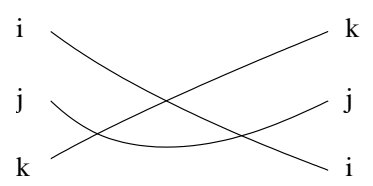

a)

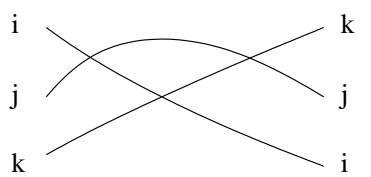

b)

Figure 4: The set $\{i, j, k\}$ is an inversion in a), but not in b)

out all the transpositions in the maximal reduced decomposition $s_{1} s_{2} s_{3} s_{1} s_{2} s_{1}$, we get the arrangement of pseudolines shown in Figure 5.

It is easy to see that, given a maximal reduced decomposition, one can construct form it an arrangement of pseudolines the way we did above. But will distinct maximal reduced decompositions result in distinct arrangements? The example above reveals that the answer must be "no." The maximal reduced decomposition $s_{1} s_{2} s_{1} s_{3} s_{2} s_{1}$ would result in exactly the same arrangement - in other words, switching the adjacent $s_{1}$ and $s_{3}$ does not change the arrangement. In general, the maximal reduced decompositions that correspond to the same arrangements of pseudolines are equivalent [8]. Thus we can represent an equivalence class of maximal reduced decompositions as an arrangement of pseudolines. It will be significant in section 3.1 that the inversion set $I \in \mathbb{B}(n, 2)$ corresponding to the equivalence class can be identified in the arrangement as follows. When restriction of the arrangement to any triple $i<j<k$ will either look like an upward pointing triangle or like a downward pointing triangle (Figure 4). In the first case $\{i, j, k\}$ is an inversion, while in the second case it is not.

Though it is implicit in the above construction, we emphasize that the permutations visited by an equivalence class of maximal reduced decompositions can be recovered from its corresponding arrangement. Each such permutation corresponds to an additional line added to the arrangement, i.e. a new line that crosses every other line exactly once. The permutation corresponding to such a line is obtained by carrying out all the transpositions that correspond to the vertices to the left of the new line. For example, the line in Figure 6 corresponds to $s_{1} s_{2} s_{1}$, i.e. to 3214 . Equivalently we could describe the permutation as the order in which the new line crosses the four original lines: first it crosses line 3, then 


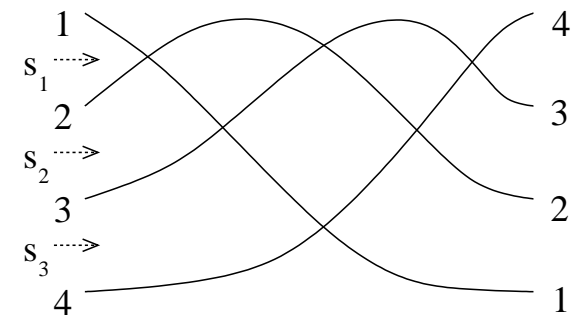

Figure 5: The arrangement of pseudolines corresponding to $s_{1} s_{2} s_{3} s_{1} s_{2} s_{1}$

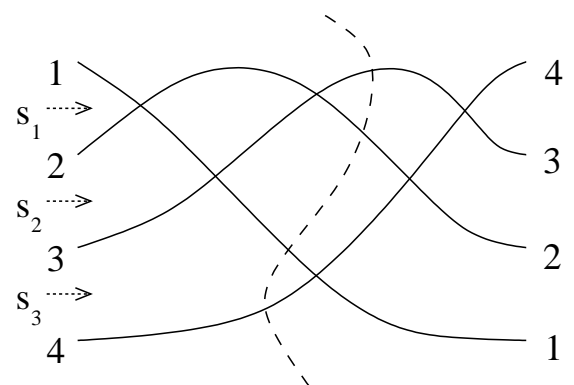

Figure 6: The permutation corresponding to this new line is 3214

line 2, line 1 and line 4 . Another way to describe this idea is through defining a "natural" partial order on the vertices of the arrangement of pseudolines.

Definition 2.6 Let $\mathcal{V}_{n}$ be the set of vertices of an arrangement of pseudolines $\mathcal{A}$ on $n$ strings. The natural partial order $P_{\mathcal{A}}$ on the vertices $\mathcal{V}_{n}$ is defined by

$$
i j P_{\mathcal{A}} k l \quad \Longleftrightarrow \quad\{i, j\} \cap\{k, l\} \neq \emptyset
$$

and $i j$ is to the left of $k l$ on the line connecting them.

For example, the arrangement in Figure 5 has $\mathcal{V}=\{12,13,14,23,24,34\}$ as its vertex set, and its natural partial order is:

$$
\begin{array}{lll}
12 & P_{\mathcal{W}} & 13 \\
13 & P_{\mathcal{W}} & 14 \\
12 & P_{\mathcal{W}} & 23 \\
23 & P_{\mathcal{W}} & 24 \\
13 & P_{\mathcal{W}} & 23 \\
23 & P_{\mathcal{W}} & 34 \\
14 & P_{\mathcal{W}} & 24 \\
24 & P_{\mathcal{W}} & 34
\end{array}
$$



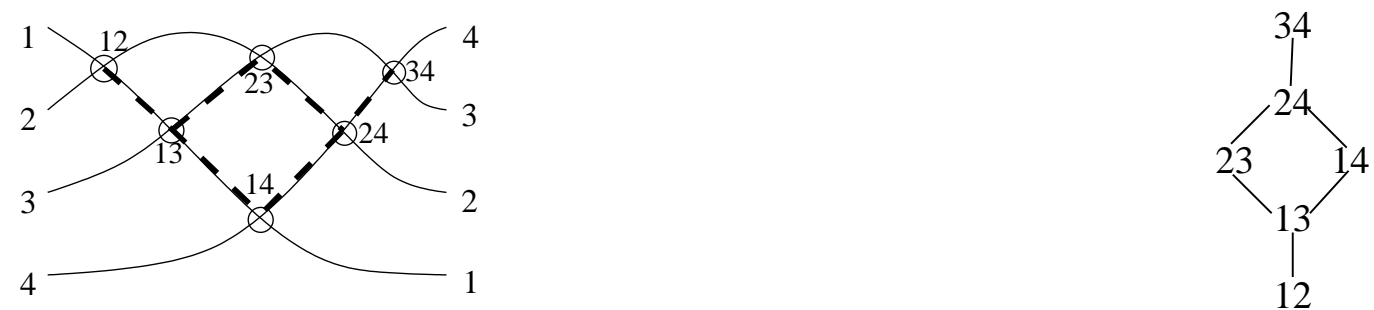

Figure 7: The natural partial order on the vertices of an arrangement

Figure 7 illustrates how the natural partial order is constructed. Drawing a new line, as above, now determines an order ideal in the natural partial order. For example, the new line drawn in Figure 6 corresponds to the ideal generated by the vertex 23. Thus, in general, the lattice of ideals of the natural partial order can be thought of as a partial order on the permutations visited by the equivalence class of maximal reduced decompositions corresponding to the arrangement. Moreover, as we show in Theorem 2, this lattice is a sublattice of the weak Bruhat order.

\section{3 "Large" acyclic sets}

Abello [1] and Fishburn [6, 7] constructed "large" acyclic sets using seemingly different approaches. We will show that both constructions are based on the same basic idea, namely that permutations visited by an equivalence class of maximal reduced decompositions form an acyclic set (Theorem 1 below). This also provides an explicit construction of the "large" acyclic sets Abello defined implicitly (Theorem 2 below). In Theorem 3 we show that Fishburn's "alternating scheme" is the set of permutations visited by the equivalence class of a particular maximal reduced decomposition, and we conjecture that it is the largest acyclic set obtainable via this construction. Before we state our results, we describe the "alternating scheme" and a useful generalization.

Fishburn [6] noted that a set of permutations is acyclic if, and only if, every triple $1 \leq i<j<k \leq n$ satisfies a never constraint of the form " $a$ is never $b$ th in the restriction 
to $\{i, j, k\}$," where $a \in\{i, j, k\}$ and $b \in\{1,2,3\} .{ }^{10}$ A never constraint is written as

$$
a N_{b} i j k .
$$

The alternating scheme is a set of such never constraints:

Definition 3.1 The alternating scheme is the following set of never constraints: ${ }^{11}$

$$
\begin{aligned}
& \text { for all } 1 \leq i<j<k \leq n, \quad j N_{3} i j k \quad \text { if } j \text { is even } \\
& j N_{1} i j k \quad \text { if } j \text { is odd. }
\end{aligned}
$$

The following generalization of the alternating scheme will be useful.

Definition 3.2 Let $U \subseteq\left(\begin{array}{c}{[n]} \\ 3\end{array}\right)$. The set of $U$-constraints is the following set of never constraints:

$$
\begin{aligned}
& \text { for all } 1 \leq i<j<k \leq n, \quad j N_{3} i j k \quad \text { if }\{i, j, k\} \notin U \\
& j N_{1} i j k \quad \text { if }\{i, j, k\} \in U \text {. }
\end{aligned}
$$

In particular, the alternating scheme is the set of $U_{A^{-}}$-constraints, where

$$
U_{A}=\{\{i, j, k\} \mid 1 \leq i<j<k \leq n \text { and } j \text { is odd }\}
$$

Every acyclic set described in this paper will satisfy a set of $U$-constraints for some $U \in \mathbb{B}(n, 2)$.

Theorem 1 The permutations visited by an equivalence class of maximal reduced decomposition form an acyclic set.

\footnotetext{
${ }^{10}$ It is easy to see that this is equivalent to acyclicity (Definition 2).

${ }^{11}$ Fishburn defines two "dually equivalent" [6] alternating schemes. For simplicity, we introduce only one of these.
} 
This result is implicit in Abello's Theorem 3.3 together with his concluding remarks $[1]$

Proof As discussed before and after Definition 2, equivalent maximal reduced decompositions correspond ${ }^{12}$ to permutations of $\left(\begin{array}{c}{[n]} \\ 2\end{array}\right)$ with the same inversion set. That is, all the maximal reduced decompositions in the equivalence class can be written as permutations of $\left(\begin{array}{c}{[n]} \\ 2\end{array}\right)$ with the same inversion set, say $I \subseteq\left(\begin{array}{c}{[n]} \\ 3\end{array}\right)$. We will show that the permutations visited by this equivalence class of maximal reduced decompositions satisfy the set of $I$ constraints. Suppose $\{i, j, k\} \in I$, with $1 \leq i<j<k \leq n$. Since $\{i, j, k\}$ is an inversion, in any maximal reduced decomposition in the equivalence class it must be that $j$ and $k$ are transposed before $i$ and $j$ are. That is, any permutation visited by the equivalence class satisfies the never constraint $j N_{1} i j k$. A similar argument shows that for any $\{i, j, k\} \notin I$, with $1 \leq i<j<k \leq n$, the never constraint $j N_{3} i j k$ is satisfied by all permutations visited by the equivalence class. Thus these permutations satisfy a set of never constraints and so they form an acyclic set.

Theorem 1 provides an explicit construction of "large" acyclic sets - but are these the maximal acyclic sets that contain a maximal chain in the weak Bruhat order? Abello showed that a maximal acyclic set that contains a maximal chain in the weak Bruhat order forms an upper semimodular sublattice of the weak Bruhat order. We show below that Abello's acyclic sets are identical with those of our Theorem 1, and they form, in fact, a distributive sublattice.

Theorem 2 Let $C$ be a maximal chain in the weak Bruhat order $\mathbb{B}(n, 1)$ (considered as a partial order on permutations). The largest acyclic set of permutations containing $C$ is the set of permutations visited by the equivalence class of the maximal reduced decomposition corresponding to $C$. This acyclic set forms a distributive sublattice of $\mathbb{B}(n, 1)$.

Proof Let $I \in \mathbb{B}(n, 2)$ be the inversion set corresponding to $C$. We have shown in Theorem 1 that the set of permutations visited by the equivalence class of the maximal

\footnotetext{
${ }^{12}$ see Figure 3
} 
reduced decomposition corresponding to $C$ form an acyclic set. In particular, all these permutations satisfy the set of $I$-constraints. The following Claim shows that no other permutation satisfies all the $I$-constraints.

Claim 1 Let $U \subseteq\left(\begin{array}{c}{[n]} \\ 3\end{array}\right)$ be an element of the higher Bruhat order $\mathbb{B}(n, 2)$ (i.e. $U$ is an inversion set). If a permutation $\pi$ of $[n]$ satisfies the set of $U$-constraints, then it is one of the permutations visited by the commuting equivalence class of maximal reduced decompositions that corresponds to $U$. That is, it is a permutation of some maximal reduced decomposition that corresponds to an admissible permutation (of $\left(\begin{array}{c}{[n]} \\ 2\end{array}\right)$ ) with inversion set $U$.

Proof By Lemma 2.2 of [16] we only need to show that the inversion set $I \subseteq\left(\begin{array}{c}{[n]} \\ 2\end{array}\right)$ of $\pi$ constitutes an ideal in the poset $Q$ that is the intersection of all admissible orders with inversion set $U$. It is easy to see that $Q$ is the transitive closure of the following relations: for all $1 \leq i<j<k \leq n$,

$$
\begin{aligned}
& \{i, j\} Q\{i, k\} \text { and }\{i, k\} Q\{j, k\} \quad \text { if } \quad\{i, j, k\} \notin U \\
& \{j, k\} Q\{i, k\} \text { and }\{i, k\} Q\{i, j\} \quad \text { if } \quad\{i, j, k\} \in U .
\end{aligned}
$$

By Lemma 2.4 in [16], for all $1 \leq i<j<k \leq n$, the inversion set of any permutation can include only an initial or a final segment of $\{i, j\},\{i, k\},\{j, k\}$. Since $\pi$ satisfies the set of $U$-constraints, its inversion set must include an initial segment of $\{i, j\},\{i, k\},\{j, k\}$ if $\{i, j, k\} \notin U$, and a final segment of $\{i, j\},\{i, k\},\{j, k\}$ if $\{i, j, k\} \in U$. Since $Q$ is defined by (14) and (15), this proves that the inversion set of $\pi$ is indeed an ideal in $Q$.

To show the second claim in the Theorem, consider the arrangement of pseudolines representing the equivalence class of maximal reduced decompositions. The permutations visited by the equivalence class correspond to ideals of the natural partial order of this arrangement. Thus the lattice of ideals is a partial order (a lattice) on these permutations. By definition, this lattice orders the inversion sets of the permutations by inclusion, so it is a sublattice of the weak Bruhat order. Since it is a lattice of ideals, by Birkhoff's Theorem 
it is distributive.

Now we show that the alternating scheme fits in the framework just described.

Theorem 3 The alternating scheme is the set of permutations visited by an equivalence class of maximal reduced decompositions.

Proof Recall that the alternating scheme is the set of $U_{A}$ constraints, where

$$
U_{A}=\{\{i, j, k\} \mid 1 \leq i<j<k \leq n \text { and } j \text { is odd }\} .
$$

We will show that $U_{A} \in \mathbb{B}(n, 2)$. In light of Theorems 1 and 2 and their proofs, this will prove the Theorem. By [16, Theorem 4.1] we need to show that for any $\{p, q, r, s\}$ with $1 \leq p<q<r<s \leq n$ the intersection of $U_{A}$ with $\{\{p, q, r\},\{p, q, s\},\{p, r, s\},\{q, r, s\}\}$ is either a beginning or an ending segment of it. If $q$ and $r$ are even, the intersection is empty; if they are both odd, the intersection is all four elements; if $q$ is odd, but $r$ even, then the intersection is the first two elements; in the symmetric case it is the last two. Thus we have verified that $U_{A} \in \mathbb{B}(n, 2)$.

We illustrate the above results with the alternating scheme for $n=4$. The arrangement of pseudolines corresponding to it is shown in Figure 8.

We can find the inversion set (and thus the element of the higher Bruhat order $\mathbb{B}(n, 2)$ ) that this arrangement corresponds to: the lines 1, 3 and 4 make a triangle that points up (Figure 9), and so $\{1,3,4\}$ is part of the inversion set corresponding to any maximal reduced decomposition represented by this arrangement. On the other hand, the lines 1,2 and 4 make a downward pointing triangle (Figure 10), and $\{1,2,4\}$ is not an inversion. The natural partial order of this wiring diagram is shown in Figure 12, and figure 11 shows it "embedded" in the arrangement.

The lattice of ideals of the natural partial order is shown in part b) of Figure 13. Part a) of Figure 13 illustrates that each ideal may be identified with the initial segment of a (non-unique) maximal reduced decomposition corresponding to the arrangement. As 


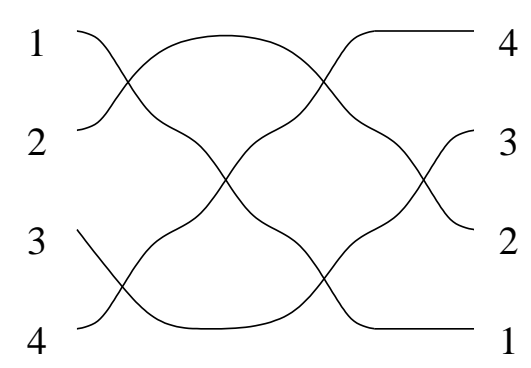

Figure 8: The alternating scheme

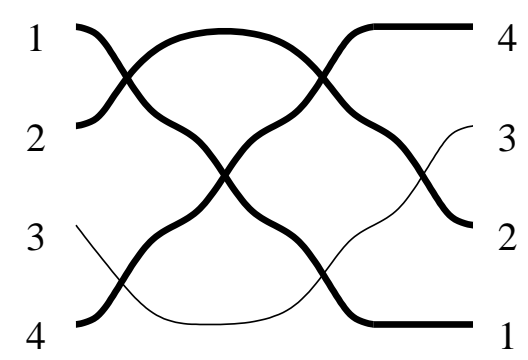

Figure 10: Strings 1,3 and 4 make a downward pointing triangle

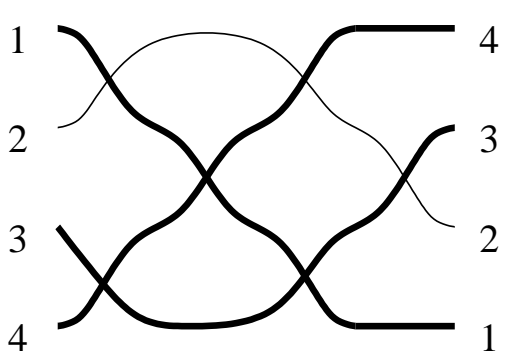

Figure 9: Strings 1,3 and 4 make an upward pointing triangle

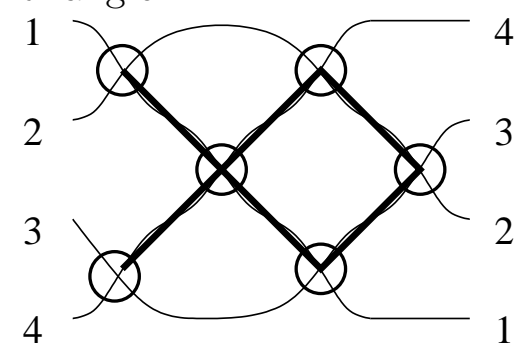

Figure 11: The natural partial order on the set of crossings 


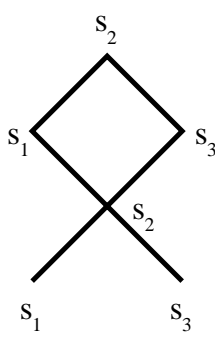

a)

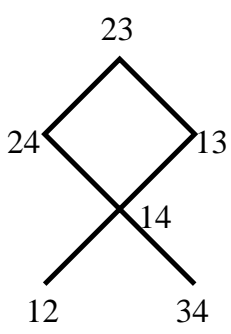

b)

Figure 12: The natural partial order

shown above, the maximal reduced decompositions identified with the top element in part a) of the Figure constitute an equivalence class. If we replace the initial segments of the maximal reduced decompositions with the permutations they generate, i.e. if we identify each element of the lattice with a permutation, we get the sublattice of the weak Bruhat order $\mathbb{B}(4,1)$ described in Theorem 2 - this sublattice is shown in part c) of Figure 13 and is highlighted in Figure 2.

\subsection{Enumerating the alternating scheme}

Fishburn conjectured [6, Conjecture 2] that among acyclic sets that do not use an $N_{2}$ (or "never second") constraint, the alternating scheme has maximum cardinality. The following conjecture is a weakening of his.

Conjecture 1 Among acyclic sets that are the permutations of some equivalence class of maximal reduced decompositions, the alternating scheme has maximum cardinality.

This conjecture is based on intuition from enumerating the alternating scheme in the particular way we describe below. Fishburn has shown that for $n \leq 6$, the alternating scheme achieves maximum cardinality, which implies the Conjecture above for those cases. We have checked that it also holds for $n=7$.

Our method of enumerating the permutations that satisfy the alternating scheme of Fishburn [7] amounts to deriving the cardinality of the lattice of ideals of the natural 


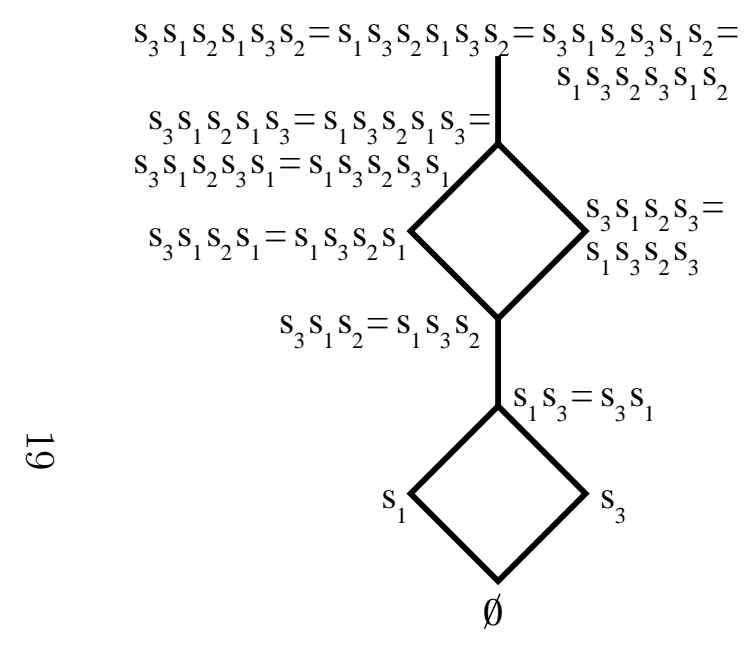

a)

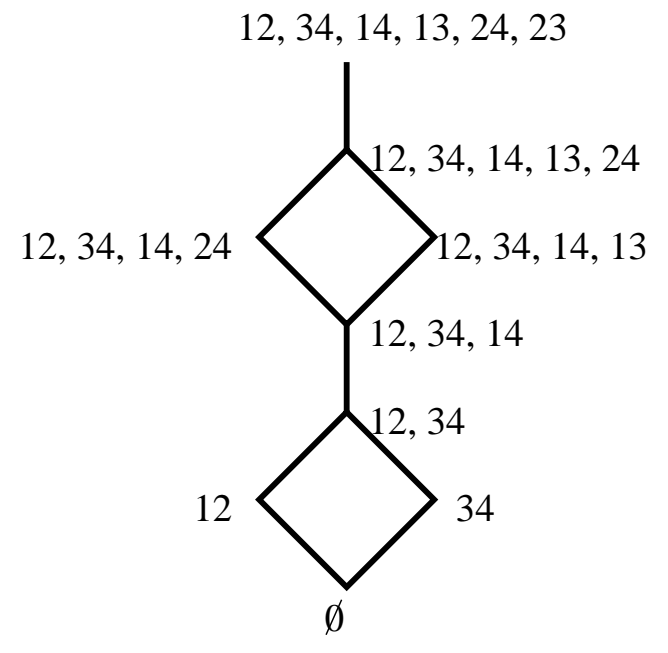

b)

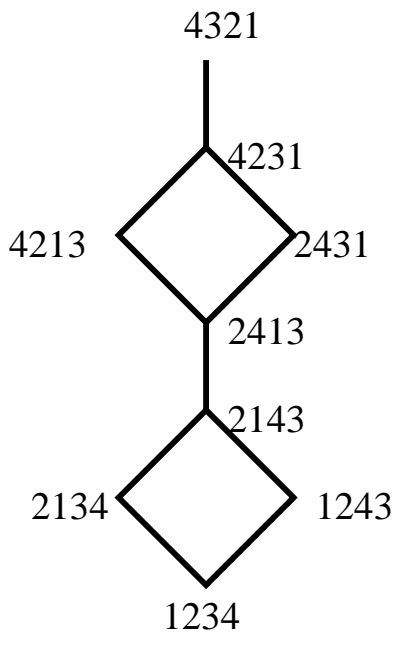

c)

Figure 13: The lattice of ideals of the natural partial order 
partial order of the arrangement of pseudolines that corresponds to the inversion sets $U_{A}$ (see (13) on p. 13). For example, the arrangement in Figure 8 corresponds to the inversion set of the alternating scheme when $n=4:\{\{1,3,4\},\{2,3,4\}\}$. Thus we can enumerate the permutations satisfying the alternating scheme by counting the ideals of the poset in Figure 12. There are nine such ideals, and, indeed, the cardinality of the alternating scheme is nine when $n=4$. Because the natural partial order of an arrangement of pseudolines corresponding to the alternating scheme is very regular, we can pursue the same strategy to derive a general formula.

We illustrate the approach for $n=8$. The arranegement corresponding to the alternating scheme is shown in Figure 14. The regularity of the diagram is not coincidental: every arrangement corresponding to the alternating scheme for even $n$ will "look the same." To see why, notice that even numbered lines must move up first, because they are in the middle of non-inversions. Then they must cross every smaller line before they cross any of the larger ones. Odd lines must move down first, because they are in the middle of inversions. Then they must cross every larger line before they cross any of the smaller ones. The natural partial order is shown in Figure 14. To enumerate the alternating scheme, we must count the ideals of this poset. An ideal can be identified by its "upper boundary," as shown in Figure 14. The empty ideal, however, cannot be represented by such a boundary, and neither can any of the ideals consisting of fewer than four of the elements in the bottom rank. To correct this, we add two extra ranks at the bottom, extending the arrangement and the natural partial order as in Figure 15. We can represent the empty ideal by the boundary shown in Figure 15. Now every ideal may be represented by its boundary, that is, by a line in the arrangement that starts on the top and proceeds downward until it reaches the bottom. In other words, every ideal corresponds to a path from one of the circled points on the top to one of those circled on the bottom (Figure 16). This means that enumerating the set of permutations that satisfy the alternating scheme amounts to counting the paths from top to bottom in the extended version of the natural partial order of the arrangement. We use standard lattice path enumeration techniques to sum these paths, and then manipulate the resulting sums to obtain the formula in the following 


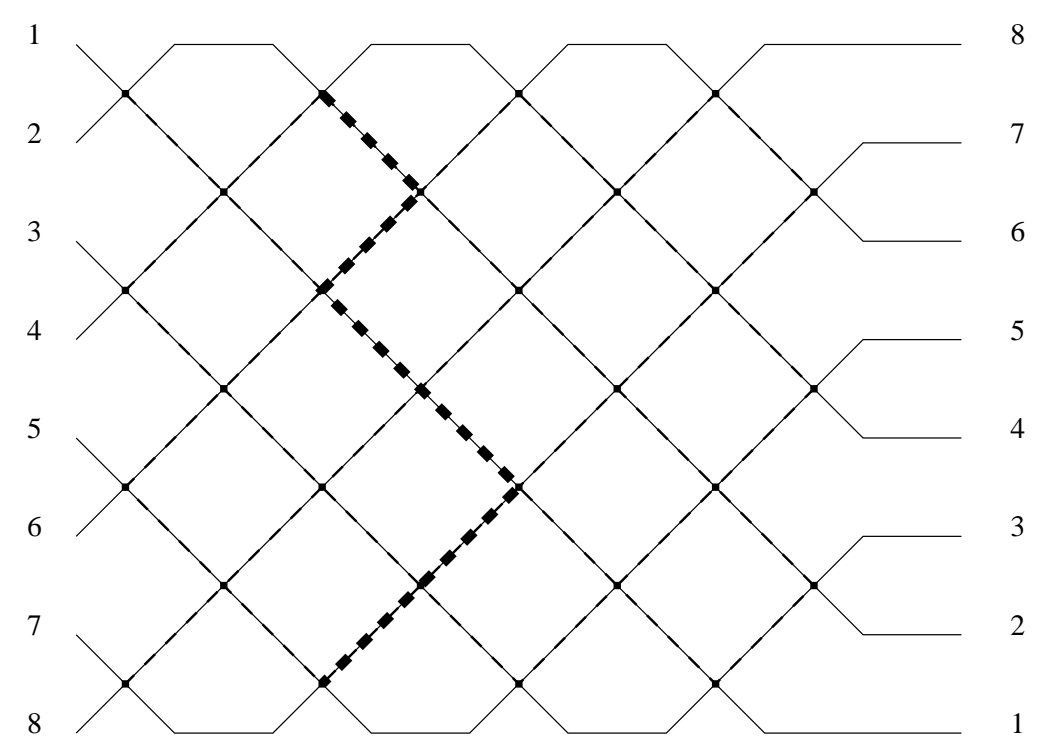

Figure 14: The arrangement for the alternating scheme when $n=8$. The natural partial order is highlighgted. An ideal can be identified by its "boundary"

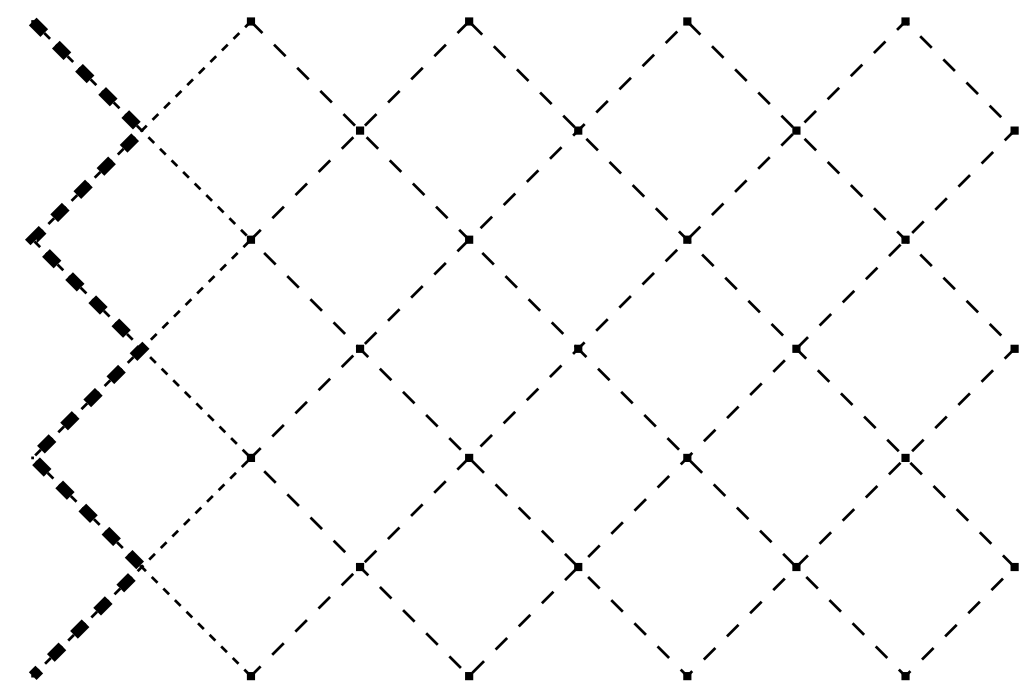

Figure 15: The natural partial order, extended so that the empty ideal can be represented as a lattice path (drawn thick) 


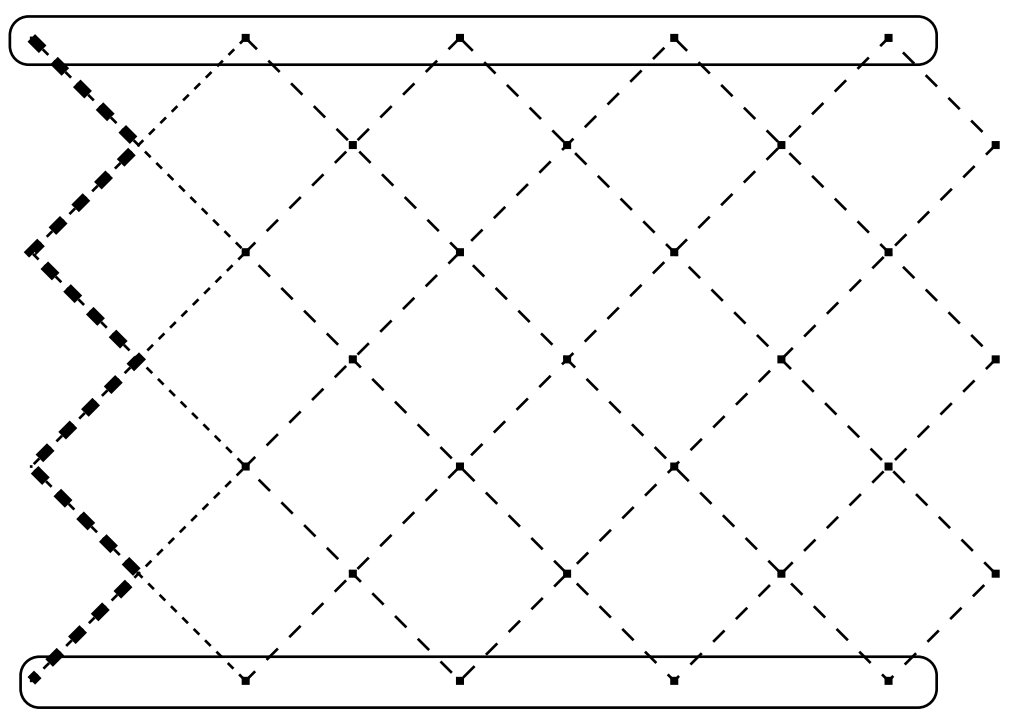

Figure 16: Representing ideals as paths

Theorem.

Theorem 4 The cardinality of the alternating scheme is

$$
A_{n}=2^{n-3}(n+3)-\left(\begin{array}{c}
n-2 \\
\frac{n}{2}-1
\end{array}\right)\left(n-\frac{3}{2}\right)
$$

for even $n$, and

$$
A_{n}=2^{n-3}(n+3)-\left(\begin{array}{c}
n-1 \\
\frac{n-1}{2}
\end{array}\right)\left(\frac{n-1}{2}\right)
$$

for odd $n$.

Proof We will prove the theorem for even $n$ - the case of odd $n$ is very similar. The extended version of the natural partial order of the arrangement for $n$ even will be just like that shown in Figure 16, with $\frac{n}{2}+1$ "circled" starting points on the top (and the same number of ending points on the bottom). The length of a path will be $n-2$. Using standard lattice path counting techniques, we get

$$
A_{n}=\sum_{i=1}^{\frac{n}{2}+1} \sum_{j=1}^{\frac{n}{2}+1}\left(\begin{array}{c}
n-2 \\
\frac{n}{2}-1+|i-j|
\end{array}\right)-\left(\begin{array}{c}
n-2 \\
\frac{n}{2}+i+j-2
\end{array}\right)-\left(\begin{array}{c}
n-2 \\
\frac{n}{2}+n-i-j+3
\end{array}\right) .
$$


We define

$$
\begin{aligned}
& A_{n}^{1}:=\sum_{i=1}^{\frac{n}{2}+1} \sum_{j=1}^{\frac{n}{2}+1}\left(\begin{array}{c}
n-2 \\
\frac{n}{2}-1+|i-j|
\end{array}\right) \\
& A_{n}^{2}:=\sum_{i=1}^{\frac{n}{2}+1} \sum_{j=1}^{\frac{n}{2}+1}\left(\begin{array}{c}
n-2 \\
\frac{n}{2}+i+j-2
\end{array}\right) \\
& A_{n}^{3}:=\sum_{i=1}^{\frac{n}{2}+1} \sum_{j=1}^{\frac{n}{2}+1}\left(\begin{array}{c}
n-2 \\
\frac{n}{2}+n-i-j+3
\end{array}\right) .
\end{aligned}
$$

$A_{n}^{1}$ counts the paths that start from the a vertex on the top and end at a vertex on the bottom. We subtract $A_{n}^{2}$ and $A_{n}^{3}$, the paths that go beyond the diagram (Figure 16) on the right or on the left. We use the reflection principle [13, p. 130] to count $A_{n}^{2}$ and $A_{n}^{3}$. Each of these can be summed using two consequences of the Binomial Theorem (for even $N)$ :

$$
\sum_{k=\frac{N}{2}+1}^{N}\left(\begin{array}{l}
N \\
k
\end{array}\right)=\frac{1}{2}\left[2^{N}-\left(\begin{array}{c}
N \\
\frac{N}{2}
\end{array}\right)\right]
$$

and

$$
\sum_{k=\frac{N}{2}+1}^{N} k\left(\begin{array}{l}
N \\
k
\end{array}\right)=\frac{N}{2} 2^{N-1} .
$$

The second of these can be derived from the "differentiated" version of the Binomial Theorem by noticing that

$$
k\left(\begin{array}{l}
N \\
k
\end{array}\right)=k \frac{N+1-k}{k}\left(\begin{array}{c}
N \\
N+1-k
\end{array}\right)=(N+1-k)\left(\begin{array}{c}
N \\
N+1-k
\end{array}\right) .
$$


We derive the formula for the first sum:

$$
\begin{aligned}
A_{n}^{1}= & \sum_{i=1}^{\frac{n}{2}+1} \sum_{j=1}^{\frac{n}{2}+1}\left(\begin{array}{c}
n-2 \\
\frac{n}{2}-1+|i-j|
\end{array}\right) \\
= & -\left(\frac{n}{2}+1\right)\left(\begin{array}{c}
n-2 \\
\frac{n}{2}-1
\end{array}\right)+\sum_{k=0}^{\frac{n}{2}} 2\left(\frac{n}{2}+1-k\right)\left(\begin{array}{c}
n-2 \\
\frac{n}{2}-1+k
\end{array}\right) \\
= & -\left(\frac{n}{2}+1\right)\left(\begin{array}{l}
n-2 \\
\frac{n}{2}-1
\end{array}\right)+\sum_{l=\frac{n}{2}+1}^{n-2} 2(n-l)\left(\begin{array}{c}
n-2 \\
l
\end{array}\right) \\
= & -\left(\frac{n}{2}+1\right)\left(\begin{array}{l}
n-2 \\
\frac{n}{2}-1
\end{array}\right)+2 n\left(\begin{array}{l}
\left.2^{n-3}+\frac{1}{2}\left(\begin{array}{c}
n-2 \\
\frac{n}{2}-1
\end{array}\right)\right)- \\
=
\end{array}\right. \\
= & 2^{n-3}(n+2)+\left(1-\frac{n}{2}\right)\left(\begin{array}{c}
n-2 \\
\frac{n}{2}-1
\end{array}\right) .
\end{aligned}
$$

Similar algebraic manipulation gives us

$$
\begin{aligned}
& A_{n}^{2}=\left(\frac{n}{4}-\frac{1}{2}\right)\left(\begin{array}{l}
n-2 \\
\frac{n}{2}-1
\end{array}\right) \\
& A_{n}^{3}=2^{n-3}\left(\frac{n}{2}-1\right)-\frac{n}{4}\left(2^{n-2}-\left(\begin{array}{c}
n-2 \\
\frac{n}{2}-1
\end{array}\right)\right) .
\end{aligned}
$$

Thus we have

$$
\begin{aligned}
A_{n} & =A_{n}^{1}-A_{n}^{2}-A_{n}^{3} \\
& =2^{n-3}(n+3)-\left(\begin{array}{c}
n-2 \\
\frac{n}{2}-1
\end{array}\right)\left(n-\frac{3}{2}\right) .
\end{aligned}
$$

\section{References}

[1] James Abello. The weak Bruhat order of $s_{\sigma}$, consistent sets, and Catalan numbers. SIAM Journal of Discrete Mathematics, 4(1):1-16, February 1991. 
[2] Anders Björner, Michel Las Vergnas, Bernd Sturmfels, Neil White, and Günter M. Ziegler. Oriented Matriods. Number 46. in Encyclopedia of Mathematics and its Applications. Cambridge University Press, 1993.

[3] C. Chameni-Nembua. Regle majoritaire et distributivite dans le permutoedre. Math. Inf. Sci. hum., 27(108):5-22, 1989.

[4] S. Felsner and H. Weil. A theorem on higher Bruhat orders. Discrete and Computational Geometry, 23(1):121-127, 2000.

[5] Stefan Felsner and Günter M. Ziegler. Zonotopes associated with higher bruhat orders. Discrete Mathematics, 241:301-312, 2001.

[6] Peter C. Fishburn. Acyclic sets of linear orders. Social Choice and Welfare, 14:113124, 1997.

[7] Peter C. Fishburn. Acyclic sets of linear orders: A progress report. Social Choice and Welfare, 19:431-447, 2002.

[8] J. E. Goodman and R. Pollack. Semispaces of configurations, cell complexes of arrangements. J. Combinatorial Theory, Ser. A, 37:257-293, 1984.

[9] Yu. I. Manin and V. V. Schechtman. Higher Bruhat orders, related to the symmetric group. Functional Analysis Applications, 20:148-150, 1986.

[10] Yu. I. Manin and V. V. Schechtman. Arrangements of hyperplanes, higher braid groups and higher Bruhat orders. In J. Coates et al., editor, Algebraic Number Theory - in honor of K. Iwasawa, volume 17 of Advanced Studies in Pure Mathematics, pages 289-308. Kinokuniya Company / Academic Press, 1989.

[11] Ran Raz. VC-dimension of sets of permutations. Combinatorica, 20(2):241-255, 2000.

[12] Amartya K. Sen. A possibility theorem on majority decisions. Econometrica, 34(2):491-499, April 1966. 
[13] Dennis Stanton and Dennis White. Constructive Combinatorics. Springer-Verlag, 1986.

[14] Benjamin Ward. Majority voting and alternative forms of public enterprises. In J. Margolis, editor, The Public Economy of Urban Communities. Johns Hopkins Press, Baltimore, 1965.

[15] Takemi Yanagimoto and Masashi Okamoto. Partial orderings of permutations and monotonicity of a rank correlation statistic. Ann. Inst. Statist. Math., 21:489-506, 1969.

[16] Günter M. Ziegler. Higher Bruhat orders and cyclic hyperplane arrangements. Topology, 32(2):259-279, 1993. 